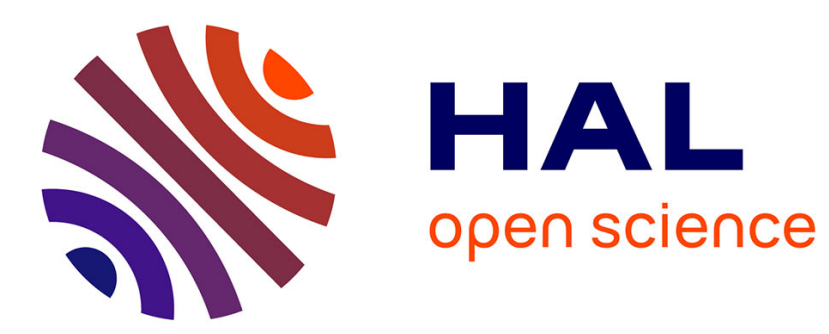

\title{
Motion Pattern for the Landing Phase of a Vertical Jump for Humanoid Robots
}

\author{
Sophie Sakka, Rio Ee Sian, Kazuhito Yokoi
}

\section{To cite this version:}

Sophie Sakka, Rio Ee Sian, Kazuhito Yokoi. Motion Pattern for the Landing Phase of a Vertical Jump for Humanoid Robots. 2006, pp.203. hal-00120461

\section{HAL Id: hal-00120461 \\ https://hal.science/hal-00120461}

Submitted on 15 Dec 2006

HAL is a multi-disciplinary open access archive for the deposit and dissemination of scientific research documents, whether they are published or not. The documents may come from teaching and research institutions in France or abroad, or from public or private research centers.
L'archive ouverte pluridisciplinaire HAL, est destinée au dépôt et à la diffusion de documents scientifiques de niveau recherche, publiés ou non, émanant des établissements d'enseignement et de recherche français ou étrangers, des laboratoires publics ou privés. 


\section{Motion Pattern for the Landing Phase of a Vertical Jump for Humanoid Robots}

\author{
Sophie Sakka \\ LMS, University of Poitiers SFA - CNRS \\ Futuroscope Chasseneuil, France \\ Email: sophie.sakka@lms.univ-poitiers.fr
}

\author{
Neo Ee Sian and Kazuhito Yokoi \\ JRL, AIST \\ Central 2, 1-1-1 Umezono, Tsukuba 305-8568, Japan \\ Email: \{rio.neo,kazuhito.yokoi\} @ aist.go.jp
}

\begin{abstract}
This paper deals with the generation of motion pattern for humanoid robots vertical jump. The study concentrates on the landing phase of the jump which is the most demanding for the system because of high discontinuity of acceleration at impact time. We first decompose the motion in two functions. The first one starts just before landing and consists in maximizing the value of the discontinuity by reducing the feet/ground velocity. We set the feet, COM and feet/COM velocities according to the supposed height of the jump. At impact time, the second function uses measured data of Ground Reaction Force (GRF) from the ankle force sensors to quickly stop the system while keeping its vertical equilibrium. The influence of each function and their respective parameters is discussed and analyzed, and their validity is tested using the model of an existing humanoid platform.
\end{abstract}

Index Terms-Humanoid robotics, vertical jump, pattern generation.

\section{INTRODUCTION}

Fast dynamics behaviors for legged robotic systems have been studied since the 1980's, starting with MIT 3D hopping machines (MIT Leg Laboratory, one-leg hopper in 1983 [12] and 3D biped in 1989 [11]). The studies issued impressive results for "stabilized" dynamic motion, i.e. repetitive jumps without tipping over. Simple dynamic laws of aerial behavior were set and the system adapted during the landing phase to insure its vertical equilibrium for the next jump. Such studies used machines aimed for jumping.

Humanoid robots are similarly classified in bipedal robotics family. A humanoid robot is a complex redundant robotic system which particularity is to be inspired from human body kinematics, or even from human appearance in some cases. These platforms appeared in the last 10 years with Honda humanoid robot in 1997. Many projects were launched since from various countries (HRP, Robo-erectus, KHR [6], GuRoo, Dav, BH1 and many others [2], [4], [8], [10]).

Humanoid robots have a large number of degrees of freedom (dof) and are usually modeled as tree structures grouping several open links chains. The tendency in reducing their weight and their size may lead to a fragile mechanical structure. Since the actuators are all together reduced in size they need reduction mechanism which generally cannot support high impact forces. Consequently it becomes challenging to design controllers able to mimic some of the human-like fast dynamics while meeting mechanical and actuators limitations.

Up-to-now, humanoid platforms have been designed for slow dynamics walking patterns. Consequently, experimental systems are limited in terms of locomotion diversity, namely the ones inducing fast dynamics. This is because of their technological design limitations. Fast dynamic gaits, for example running [14] or jumping [9], [13] or jumping for running [12], [7], [5], are still actively under investigation and spectacular results have recently been produced with ASIMO running [14]. Dynamic equilibrium unbalance approaches for full body pattern motion definitions are still being formulated, even if some recent results for new mobility patterns tend to get closer to the robot limits for dynamic balance stability [1], [3] and therefore allow new mobility patterns.

Our study proposes the implementation of a vertical jump pattern with low landing Ground Reaction Force (GRF) for humanoid robots. The propulsive phase of the jumping motion was studied in [13]. In this paper, we focus on the landing phase of the jump, assuming the characteristics of the aerial phase are known. Our approach uses two specific functions : the first one is based on landing prevision time. It is aimed to reducing the GRF impulse at landing time by reducing the relative feet/ground velocity. As it is difficult to know precisely the state of the system during the aerial phase, the second aim of the pre-landing function is to collect information for the next stage of the jump. The second function is used to bring the system to a stop while maintaining is vertical equilibrium. The basics of our study is exposed in section 2, detailing the frame of work. The two functions for landing are exposed in section 3. In section 4, the methodology for landing is simulated using the model of existing mechanical structure HRP-2. We first describe the simulation environment and then expose and discuss the results of the landing procedure.

\section{APPROACH SETTING}

\section{A. Hypothesis}

The robotic system is considered as a flying system interacting with its environment. The interaction is characterized 
by the values of the GRF given by the ankle force sensors. Our study concentrates on the landing phase of a vertical jump. We consider as a starting point that the robot has taken off in good conditions of jump, i.e. is falling vertically from a desired height of jump $h_{d}$. We also consider that there can be small uncertainties on "known" data, such as COM take-off velocity (i.e. height of jump) or non zero momentum during the aerial phase (i.e. the system can slightly turn around its center of mass). The motion is assumed as symmetric : both right and left sides actuators are moving the same way.

\section{B. Free flight motion}

There is no possibility to control the system during the aerial phase. The evolution of the COM frame during flight, according to the reference (ground) frame is described by its translation and rotation parameters grouped in the situation vector $\mathbf{X}_{G}(t)$ :

$$
\mathbf{X}_{G}=\left[\begin{array}{llllll}
x & y & z & \ell & m & n
\end{array}\right]^{t}
$$

Using the hypothesis of only vertical motion of the COM during flight, the study in this paper only considers vertical translation parameters. However, small variations of any component of the situation vector are to be taken into account before safely considering the possibility of experimentation, as discussed in the last section.

Let $\dot{\mathbf{X}}_{G}(t)$ and $\ddot{\mathbf{X}}_{G}(t)$ denote respectively the evolution of speed and acceleration with time. For the vertical jump, all the coordinates are set at zero except the cote and its derivatives which are subject to the gravity acceleration $g=9.81 \mathrm{~m} \cdot \mathrm{s}^{-2}$ :

$$
\begin{aligned}
& \ddot{z}_{G}(t)=-g \\
& \dot{z}_{G}(t)=-g t+V_{t o} \\
& z_{G}(t)=-\frac{1}{2} g t^{2}+V_{t o} t+z_{t o}
\end{aligned}
$$

where $V_{t o}=\dot{z}_{G}\left(t_{t o}\right)$ is the COM velocity at take-off time $t_{t o}$ and $z_{t o}=z_{G}\left(t_{t o}\right)$. The conservation of energy between takeoff and landing instants, and the immobility of the system during the aerial phase (i.e. altitude of the COM at take off $z_{t o}$ and at landing $z_{l d}$ are equal) lead to :

$$
\frac{1}{2} m\left(V_{l d}^{2}-V_{t o}^{2}\right)=m g\left(z_{l d}-z_{t o}\right)=0
$$

In this case, the landing velocity is known as

$$
V_{l d}=-V_{t o}
$$

and the flight time $t_{f l}=t_{l d}-t_{t o}$ is deduced from Eq. 2 :

$$
t_{f l}=\frac{2 V_{t o}}{g}
$$

Then, if the take off velocity is known precisely, the landing can be forecast and dealt with.

\section{Decomposition of motion}

The definition of impulse comes from the integration of the dynamics equations of the system

$$
m \ddot{\mathbf{X}}_{G}=m \mathbf{g}+\mathbf{R}
$$

with $\mathbf{R}=\mathbf{0}$ during the aerial phase and $\mathbf{R}=-m \mathbf{g}$ when the robot is standing. Counter-balancing the discontinuity of the GRF at take-off and landing means creating an equivalent discontinuity in COM acceleration $\ddot{\mathbf{X}}_{G}$, which is not convenient as the system has some limitations. Integration of Eq. 5 with time leads to the following equation :

$$
V(t+\delta t)-V(t)=\int_{t}^{t+\delta t}\left(g+\frac{R}{m}\right) \mathrm{dt}
$$

The GRF impulse is defined as the integral of the GRF over time, just before and after impact :

$$
I=\int_{t^{-}}^{t^{+}} R \mathrm{dt}
$$

Equation 6 shows the direct relation between the GRF impulse at landing time and relative feet/ground velocity. As an example, if the system remains immobile and is brought to full stop in 1 ts (one time step, 0.005 [s]) from a $5 \mathrm{~cm}$ jump ( $V_{t o}=1 \mathrm{~m} / \mathrm{s}$ ), this would lead to a GRF impulse of about 20 times the weight.

The ideal case is when the relative feet/ground velocity is exactly equal to zero. The common approach consists in controlling the trajectories of the legs actuators to obtain a feet/ground velocity equal to zero at landing instant. Such approach is based on the knowledge issued from bodies aerial behavior described ahead. In such case two undesired situation could happen :

- The height of jump is slightly lower than expected. Then the aerial phase is shorter in time and the system hits the ground at full velocity before motion;

- The height of jump is slightly greater than expected. Then the motion starts before the contact with the ground. The feet stay at the same altitude (relative feet/ground velocity equal to zero) until retraction is not possible any more. The system hits the ground at full velocity.

Our approach separates the landing motion in two functions : the first one takes effect before landing until contact is detected. It is called the pre-landing function, and it is aimed to reduce the feet/ground relative velocity and minimize the GRF impulse at the instant of landing. The second function takes effects when contact is detected until the system motion ends. It is called the landing function and it is aimed to stop the system rapidly while keeping GRF low and maintaining vertical equilibrium. This function uses data collected from the pre-landing function on the real landing state of the system. The two functions are described in the next section. 


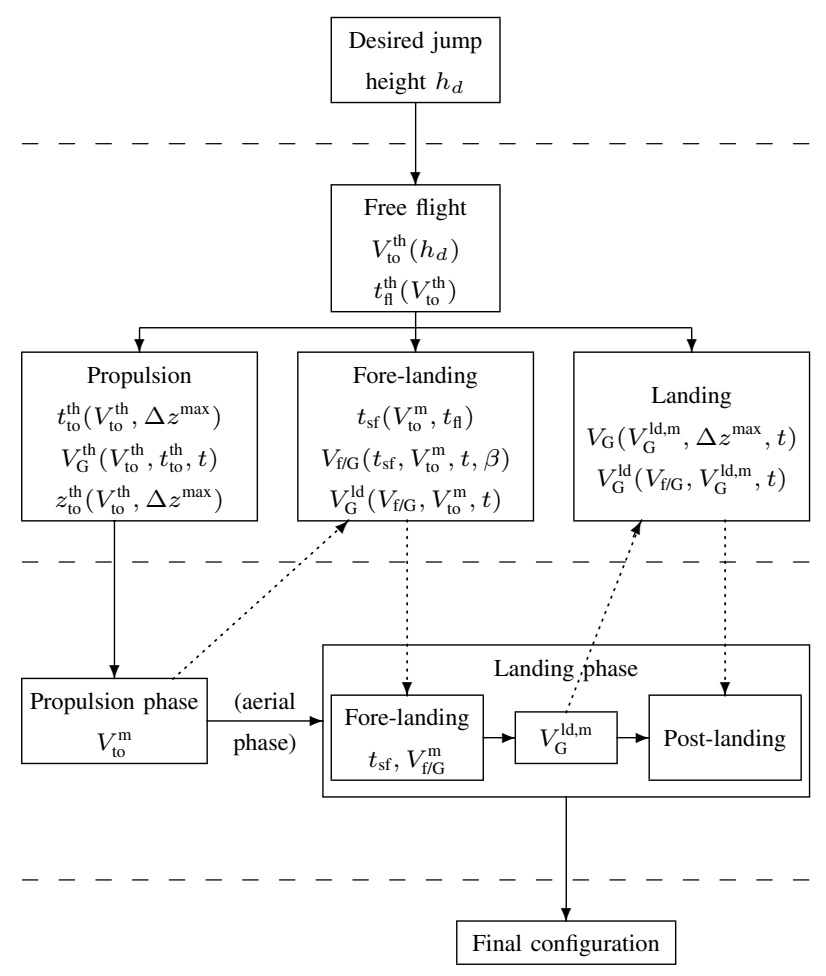

Fig. 1. General algorithm for vertical jump.

The general algorithm for the jump is shown in Fig. 1 . On this algorithm, the index $t h$ is used for theoretical values of parameter, i.e. values extracted from free flight equations (Eq. 2). The index $m$ indicates the value comes from system data. to, $f l$ and $l d$ are respectively for take-off, flight and landing. We will explicit all the mentioned parameters in the next section. The parameters exchanged between functions during the motion are noted with dotted arrows. They consist in using system state instead of state forecast by theory. The parameters are extracted while the system is in motion, and sent to functions for new calculations.

\section{FUnCTIONS DESCRIPTION}

\section{A. pre-landing function}

Figure 2 shows the notation for composed trajectories and velocities. The position of the COM can be expressed with the absolute position of the feet and their relative position to the COM. We can also express the absolute feet/ground velocity as the composition of absolute velocity of the COM and relative feet/COM velocity (Fig. 2) :

$$
V_{\mathrm{f}}^{\mathrm{th}}=V_{G}^{\mathrm{th}}-V_{\mathrm{f} / \mathrm{G}}^{\mathrm{th}}
$$

$\begin{array}{lll}\text { Absolute COM velocity : } & V_{G}^{\text {th }}(t) & \text { Estimated } \\ \text { Relative feet/COM velocity : } & V_{\text {f }}^{\text {th }}(t) & \text { Controlled } \\ \text { Absolute feet velocity : } & V_{\mathrm{f}}^{\text {th }}(t) & \text { Desired }\end{array}$

In Eq. $7, V_{G}^{\text {th }}(t)$ is the theoretical COM velocity during

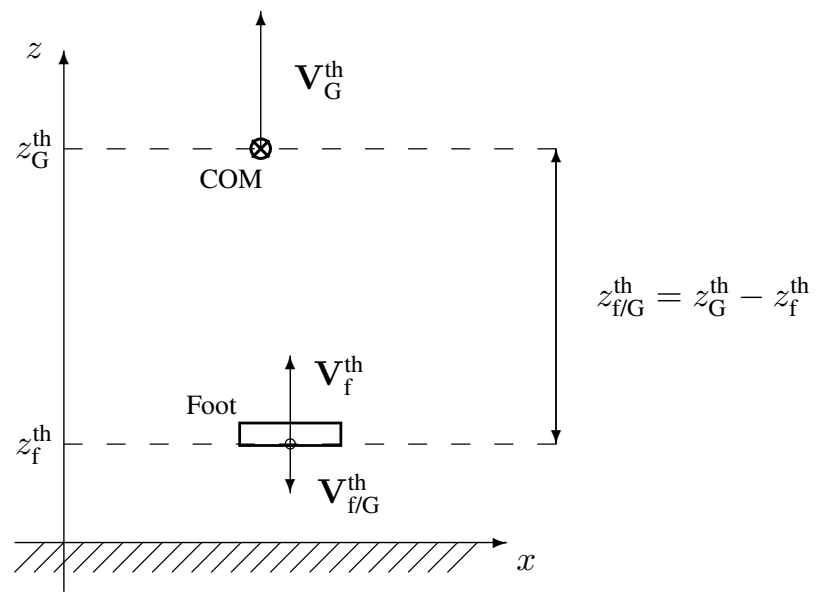

Fig. 2. Notations for composed velocities and trajectories.

flight phase, which general expression is given in system of Eqs. 2. As previously mentioned, it cannot be controlled but only estimated. Its precise expression using measured data at take-off instant is :

$$
V_{G}^{\mathrm{th}}(t)=-g\left(t-t_{\mathrm{to}}\right)+V_{\mathrm{to}}^{\mathrm{m}}
$$

$V_{\mathrm{f}}^{\text {th }}$ is the absolute feet velocity which is desired closed to zero to reduce the impact force at landing. If the system remains immobile during the aerial phase, the absolute feet velocity at landing is equal to take-off velocity (Eq. 3) :

$$
V_{\mathrm{f}}^{\mathrm{th}}\left(t_{\mathrm{ld}}\right)=-V_{\mathrm{to}}^{\mathrm{m}}
$$

Aiming for an exact feet/ground zero velocity at landing time is dangerous because of numerous uncertainties of the system. Therefore we introduce a parameter $\alpha<<0.1$ such as

$$
\lim _{t \rightarrow \infty} V_{\mathrm{f}}^{\mathrm{th}}(t)=-\alpha V_{\mathrm{to}}^{\mathrm{m}}
$$

where $\alpha$ is close to zero but non equal to zero so that the feet/ground velocity consequently reduces but has no chance to reach zero during flight phase. In such case, even if landing is delayed because of perturbations, the feet cannot go up faster than the robot goes down. We choose an exponential profile for the feet velocity such as

$$
V_{\mathrm{f}}^{\mathrm{th}}(t)=-\left(g\left(t_{\mathrm{sf}}-t_{\mathrm{to}}\right)-(1+\alpha) V_{\mathrm{to}}^{\mathrm{m}}\right) e^{-\frac{\left(t-t_{\mathrm{sf}}\right)}{\tau}}-\alpha V_{\mathrm{to}}^{\mathrm{m}}
$$

where $t_{\mathrm{sf}} \in\left[t_{\mathrm{to}}, t_{\mathrm{ld}}\right]$ is the starting time of the pre-landing function. It is set according to the flight time of the jump and a parameter $\beta$ :

$$
t_{\mathrm{sf}}=\beta *\left(t_{\mathrm{ld}}^{\mathrm{th}}-t_{\mathrm{to}}\right)+t_{\mathrm{to}}
$$

The parameter $\tau$ influences the rapidity of convergence of the exponential function. Some example using different values are shown in Fig. 3 and 4. The boundary conditions in Eq. 10 are set according to COM velocity continuity :

$$
V_{\mathrm{f}}^{\mathrm{th}}\left(t_{\mathrm{sf}}\right)=V_{G}^{\mathrm{th}}\left(t_{\mathrm{sf}}\right)=-g\left(t_{\mathrm{sf}}-t_{\mathrm{to}}\right)+V_{\mathrm{to}}
$$




$$
\lim _{t \rightarrow \infty} V_{\mathrm{f}}^{\mathrm{th}}(t)=-\alpha V_{\mathrm{to}}^{\mathrm{m}}
$$

Using at time $t=t_{\mathrm{sf}}$ the free flight COM velocity defined in Eq. 8. Eq. 10 is illustrated in Fig. 3(a). The influence of the three parameters $\alpha, \beta$ and $\tau$ can be observed : $\alpha$ sets the boundary of the damping function; $\beta$ sets its starting time and $\tau$ influences its fastness. We can also notice in Fig. 3(a) that the observed landing time $t_{\mathrm{ld}}^{\mathrm{m}}$ happens later than the forecast. This is due to the effects of the pre-landing function, which principle consists in retracting the feet slightly before landing. Then the aerial phase gets longer, and the new landing time is given using the equation of the feet trajectory (Fig.4(a)) :

$$
z_{\mathrm{f}}^{\mathrm{th}}(t)=-A \tau\left(1-e^{-\frac{t-t_{\mathrm{sf}}}{\tau}}\right)-\alpha V_{\mathrm{to}}^{\mathrm{m}}\left(t-t_{\mathrm{sf}}\right)+K_{1}
$$

with $A$ defining the velocity amplitude :

$$
A=g\left(t_{\mathrm{sf}}-t_{\mathrm{to}}\right)-(1+\alpha) V_{\mathrm{to}}^{\mathrm{m}}
$$

and

$$
K_{1}=-\frac{1}{2} g\left(t_{\mathrm{sf}}-t_{\mathrm{to}}\right)^{2}+V_{\mathrm{to}}^{\mathrm{m}}\left(t_{\mathrm{sf}}-t_{\mathrm{to}}\right)
$$

Controlled feet/COM velocity is obtained by reporting the absolute feet velocity profile of Eq. 10 in Eq. 7:

$$
\begin{gathered}
V_{\mathrm{f} / \mathrm{G}}^{\mathrm{th}}(t)=-A\left(1-e^{-\frac{\left(t-t_{\mathrm{sf}}\right)}{\tau}}\right)-g\left(t-t_{\mathrm{sf}}\right) \\
z_{\mathrm{f} / \mathrm{G}}^{\mathrm{th}}(t)=-A\left(\left(t-t_{\mathrm{sf}}\right)-\tau\left(1-e^{-\frac{\left(t-t_{\mathrm{sf}}\right)}{\tau}}\right)\right)-\frac{1}{2} g\left(t-t_{\mathrm{sf}}\right)^{2}+z_{\mathrm{to}}
\end{gathered}
$$

\section{B. Landing function}

The pre-landing function was defined to damp GRF impulse while forecasting the landing. Once the feet are on the ground, we define the landing function which aim is to bring the system to a stop. We consider two sets of constraints :

- mechanical constraints;

- maintenance of equilibrium.

Maintenance of equilibrium is insured by allowing only vertical motion of the COM. Mechanical constraints mainly consider the respect of leg amplitude and actuation capacities. We consider a simple cosine function for the controlled feet/COM relative velocity and express the continuity of the velocity at impact instant $t_{\mathrm{ld}}^{\mathrm{m}}$ and at stopping time $t_{\mathrm{e}}$ :

$$
\begin{aligned}
& V_{\mathrm{f} / \mathrm{G}}^{\mathrm{th}}(t)=\frac{1}{2}\left[1+\cos \left(\frac{2 \pi}{T}\left(t-t_{\mathrm{ld}}^{\mathrm{m}}\right)\right)\right] V_{\mathrm{f} / \mathrm{G}}^{\mathrm{th}}\left(t_{\mathrm{ld}}^{\mathrm{m}}\right) \quad t \in\left[t_{\mathrm{ld}}^{\mathrm{m}}, t_{\mathrm{e}}\right] \\
& =\quad 0 \quad t>t_{\mathrm{e}}
\end{aligned}
$$

This function is illustrated in Fig. 5(a), as well as the COM velocity during landing. A discontinuity in COM velocity can be observed on the graph at time $t_{\mathrm{ld}}^{\mathrm{m}}$. After landing, both COM velocity and feet/COM velocity follow the same profile. The value of the discontinuity is the value of the feet speed at landing time :

$$
V_{\mathrm{f}}^{\mathrm{th}}\left(t_{\mathrm{ld}}^{\mathrm{m}}\right)=-A e^{-\frac{\left(t_{l d}^{m}-t_{\mathrm{sf}}\right)}{\tau}}-\alpha V_{\mathrm{to}}^{\mathrm{m}}
$$

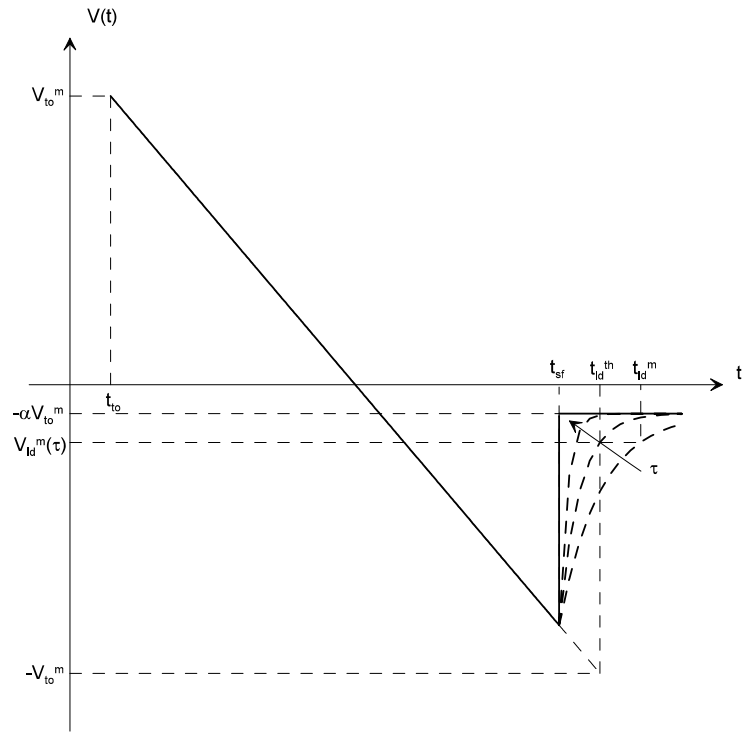

(a) Absolute velocity : $V_{\mathrm{f}}^{\text {th }}(t)$

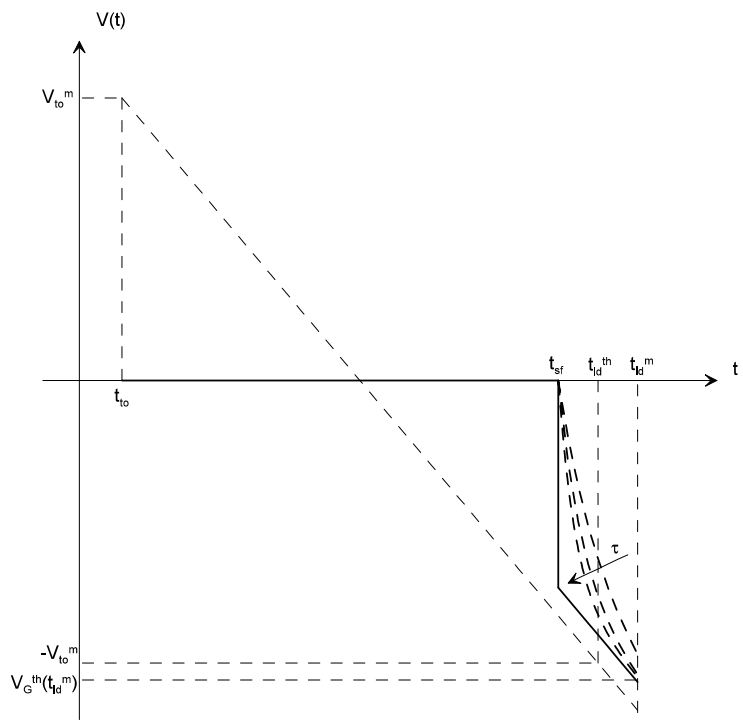

(b) Relative velocity $: V_{\mathrm{f} / \mathrm{G}}^{\mathrm{th}}(t)$

Fig. 3. Feet velocities during pre-landing function.

The stopping time is determined for $V_{\mathrm{f} / \mathrm{G}}^{\text {th }}(t)=0$, i.e. $\left(t_{\mathrm{e}}-\right.$ $\left.t_{\mathrm{ld}}^{\mathrm{m}}\right)=\frac{T}{2}$. The value of the period is constrained by two conditions :

- Maximal capacities of the actuators : fix the maximal landing speed the robot can handle in good conditions;

- Geometric capacities of the robot (minimal position of COM while in standing position).

These constraints are detailed thereafter. The stopping COM trajectory is, for $t \in\left[t_{\mathrm{ld}}^{\mathrm{m}}, t_{\mathrm{e}}\right]$ :

$$
z_{G}^{\mathrm{th}}(t)=\frac{1}{2} V_{\mathrm{f} / \mathrm{G}}^{\mathrm{th}}\left(t_{\mathrm{ld}}^{\mathrm{m}}\right) *\left[\left(t-t_{\mathrm{ld}}^{\mathrm{m}}\right)-\frac{T}{2 \pi} \sin \left(\frac{2 \pi}{T}\left(t-t_{\mathrm{ld}}^{\mathrm{m}}\right)\right)\right]+z_{\mathrm{ld}}^{\mathrm{m}}
$$




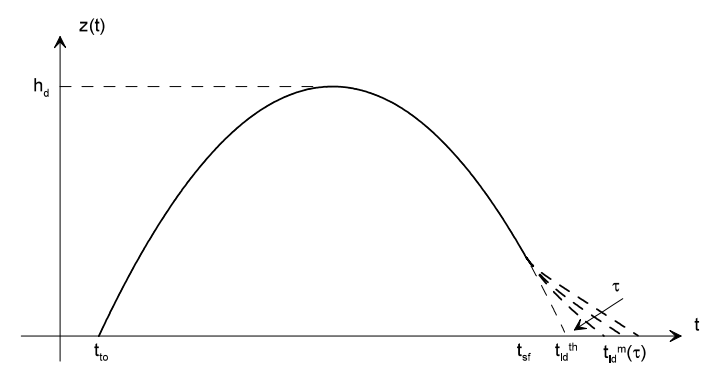

(a) Absolute trajectory : $z_{\mathrm{f}}^{\text {th }}(t)$

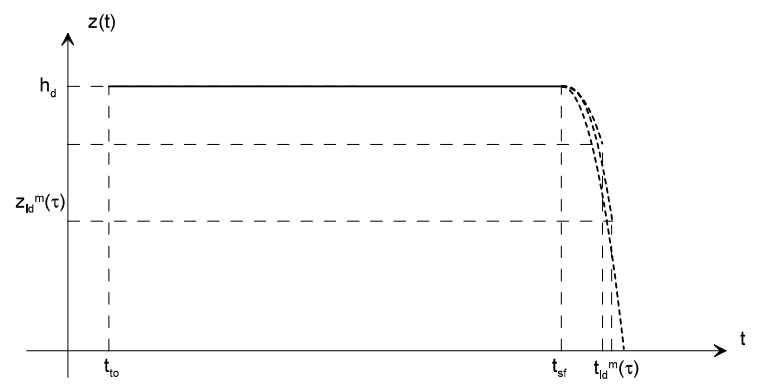

(b) Relative trajectory : $z_{\mathrm{f} / \mathrm{G}}^{\text {th }}(t)$

Fig. 4. Feet trajectories during pre-landing function.

The geometric constraint can be expressed using the final COM altitude

$$
z_{G}^{\mathrm{th}}\left(t_{\mathrm{e}}\right)=\frac{1}{2} V_{G}^{\mathrm{th}}\left(t_{\mathrm{ld}}^{\mathrm{m}}\right) *\left[\left(t_{\mathrm{e}}-t_{\mathrm{ld}}^{\mathrm{m}}\right)-\frac{T}{2 \pi}\right]+z_{\mathrm{ld}}^{\mathrm{m}} \geq z_{G}^{\min }
$$

where $z_{G}^{\min }$ is the minimal altitude admissible for the COM.

$$
\begin{gathered}
\frac{1}{2} V_{G}^{\mathrm{th}}\left(t_{\mathrm{ld}}^{\mathrm{m}}\right) *\left[1-\frac{1}{\pi}\right] \frac{T}{2}+z_{\mathrm{ld}}^{\mathrm{m}} \geq z_{G}^{\mathrm{min}} \\
\Rightarrow T \leq \frac{4 \pi\left(z_{G}^{\mathrm{min}}-z_{\mathrm{ld}}^{\mathrm{m}}\right)}{(\pi-1) V_{G}^{\mathrm{th}}\left(t_{\mathrm{ld}}^{\mathrm{m}}\right)}
\end{gathered}
$$

This inequality shows that if the motion amplitude possibilities are too few or if the landing velocity is too great, the period has to be lowered. For simulation, the value of the period is fixed to $1 \mathrm{~s}$. If the constraint expressed in Eq. 22 is not respected, then the period is equal to the right side of the inequality.

\section{Simulation RESUlts}

The two functions described in the previous section were implemented using OpenHRP simulator, using the values of the parameters given in table I. The simulation uses the model of HRP-2 humanoid robot. The simulation environment translates operational velocities into generalized velocities and sends them to control [13]. For jumping motion, the trajectories of hip, knees and ankle pitch joints are shown in Fig. 6, on which the different phases for the complete jump appear :

- Propulsion phase, from 400 ts to 830 ts;

- Aerial phase, from 830 ts to 870 ts;

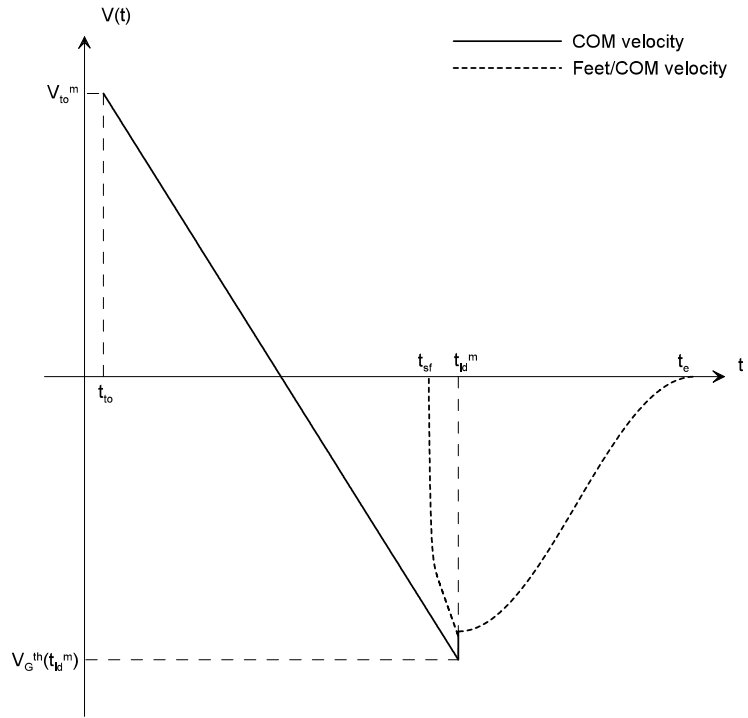

(a) Velocity

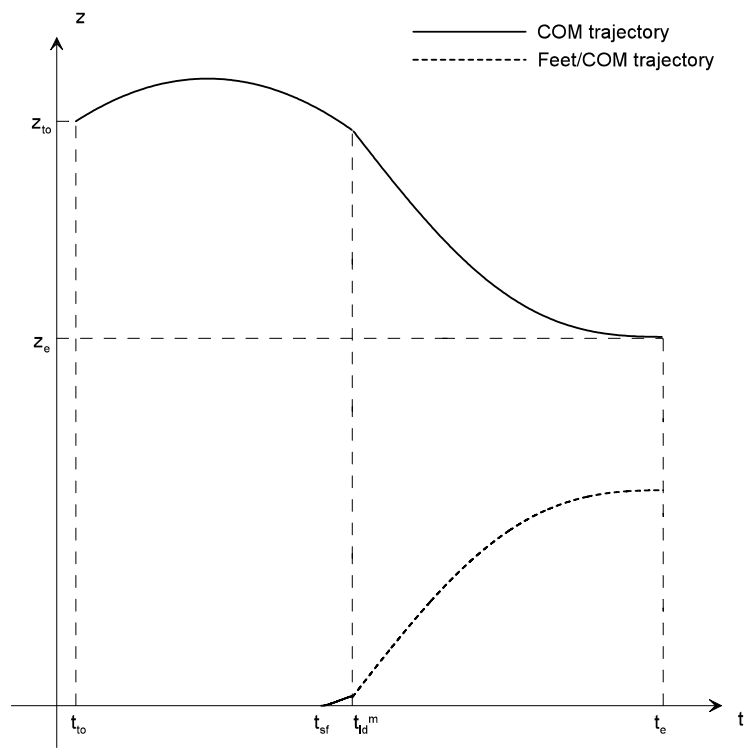

(b) Trajectory

Fig. 5. Landing COM and feet/COM velocities and trajectories.

- landing phase, from 870 ts to 920 ts.

Where ts denotes time step of control loop, 0.005 seconds. These joint trajectories illustrate the motion using both prelanding and landing functions. We discuss in this section the GRF value with time during the landing phase for a vertical jump of $10 \mathrm{~cm}$ high. Fig. 7 compares the initial signal from the $z$-axis (vertical axis) of the sensor data to the signal obtained using only the pre-landing function. Sensor data for each foot are shown. They are mainly identical but some slight differences can appear between left and right foot. The general aspect of the graphs show the propulsion, aerial $(\mathrm{GRF}=0 \mathrm{~N})$ and landing phase. The motion is considered as finished when 
TABLE I

FUNCTIONS PARAMETERS

\begin{tabular}{|l|c|c|c|c|}
\hline Parameter & $\alpha$ & $\beta$ & $\tau$ & $T$ \\
\hline Value & 0.01 & 0.9 & 0.01 & $1[\mathrm{~s}]$ \\
\hline
\end{tabular}

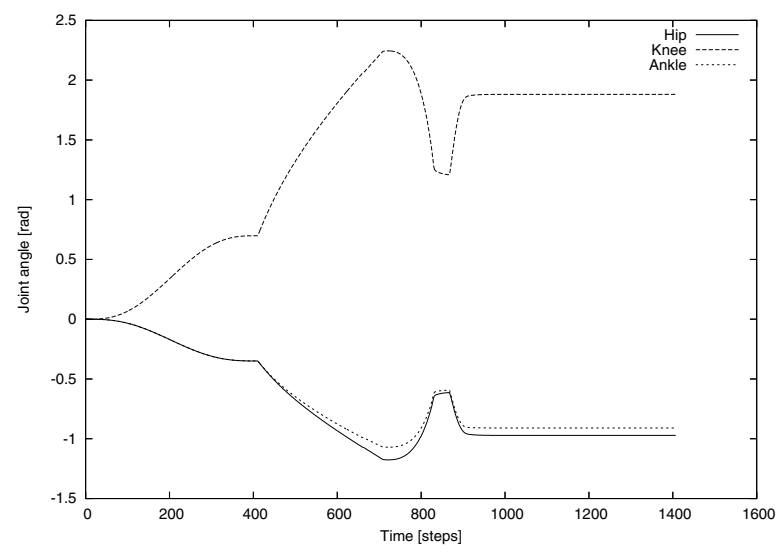

Fig. 6. Pitch angles of leg joints during jump motion pattern.

the signal is stabilized at the constant value of $290 \mathrm{~N}$ (half of the weight on each foot).

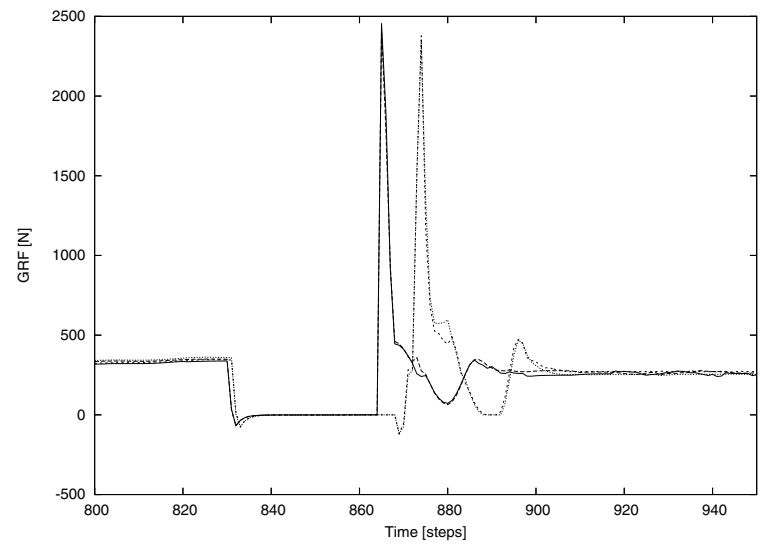

Fig. 7. Evolution of GRF with time, data from ankle force sensors. The first peak (continuous line) shows the initial signal (right and left feet). The second peak (dotted line) shows the delay of landing when using the prelanding function with $\beta=0.9$.

The initial signal (first peak in Fig. 7) is obtained using exact theory values extracted from the behavior of rigid bodies (section II.B). Because of a difference between expected values and simulated ones, a large impulse appears after the aerial phase. It shows a full velocity impact when the feet reach the ground. This is created by a delay in landing motion. After impact, the landing trajectory is respected and brings the system to a stop. The second peak in Fig. 7 represented by two dotted lines (left and right foot) shows the delay in landing created by the effects of the pre-landing function. A first impulse appears around $260 \mathrm{~N}$ at time $t=870$ ts. The parameter $\beta$ was set to 0.9 and deals with the uncertainties on the landing time prevision. A lower value for this parameter does not allow to keep robot's vertical equilibrium and a higher value does not reduce consequently the feet velocity. The second (main) impulse matches the immobility of the system as soon as the contact is detected. For the main impulse, the values of the two cases are similar $(2500 \mathrm{~N})$, which clearly show that the pre-landing function does not reduce the COM velocity. This function mainly grants some reaction time as contact impulse is too violent to be handled when detected. This function is "only" a preparation for the main landing functions to take place in good conditions.

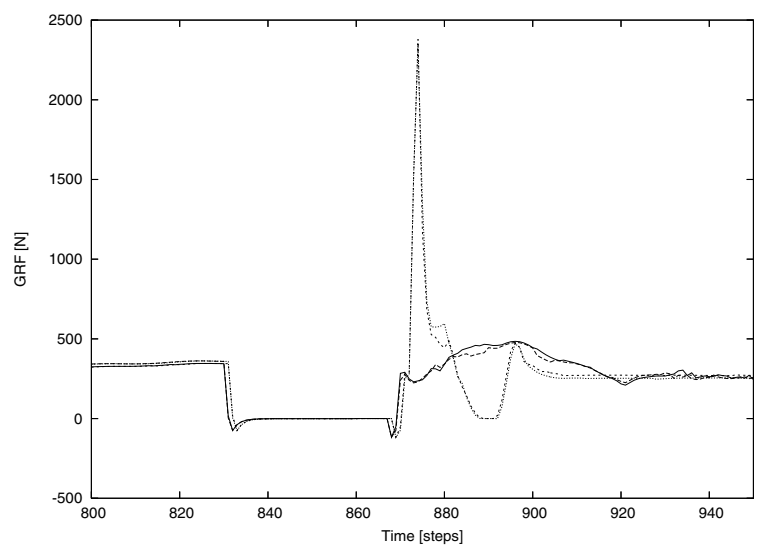

Fig. 8. Evolution of GRF with time, data from ankle force sensors. The peak (dotted line) results from using the pre-landing function alone (right and left feet). The continuous line results from using both pre-landing and landing functions. Only the compounding of the two functions allows substantial reduction of GRF when landing.

Figure 8 compares the complete landing motion pattern (pre-landing and landing functions) with the previous result using only pre-landing function. The impulse appears clearly and reaction force does not exceed $500 \mathrm{~N}$. The main impulse at $2500 \mathrm{~N}$ is resolved by damping as the landing function uses measured foot velocity at landing and adapts the pattern to the actual state.

This approach showed good results for small variations on landing time. If landing occurs within a range of $+/-3$ ts (7.5\% of aerial phase) around expected landing time, the GRF remain under $900 \mathrm{~N}$. On the other hand, if an error occurs on the momentum and creates a small rotation around the COM during the aerial phase, the torque data are out of the force sensors range of use. Figures 9(a) and 9(b) shows the data registered by the sensors through the whole motion, respectively force and torque, for such case. We can observe that the vertical GRF at landing (Fig. 9(a)) is within "reasonable" values. Figure 9(b) shows clearly that the robot landed on its toes and went on oscillating forth and back while standing. 


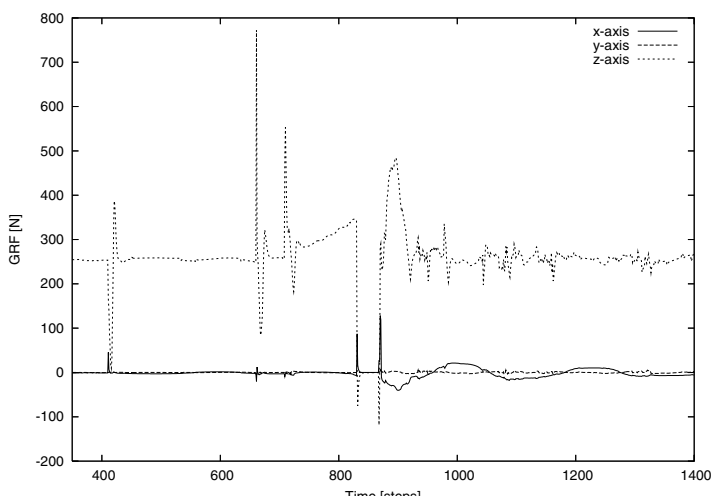

(a) Fime [steps]

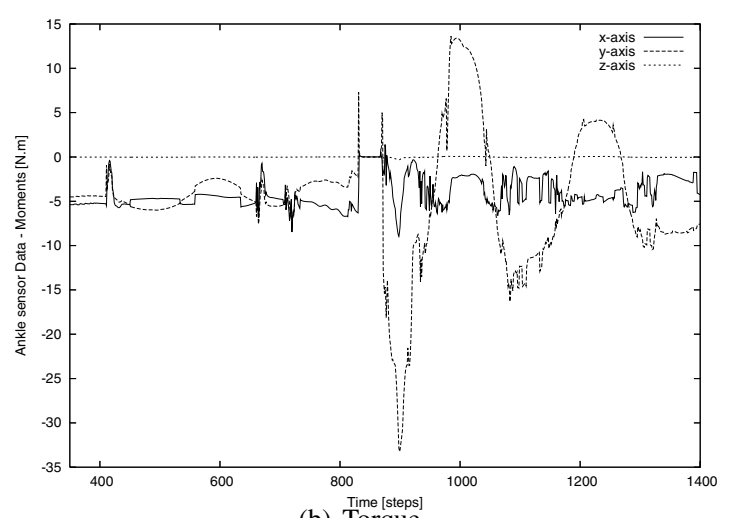

(b) Torque

Fig. 9. Ankle sensor data for the complete vertical jump. The oscillations of the robot after landing can be observed through the linear reaction force data (x-axis force from (a)) and angular reaction force (b).

\section{CONCLUSION AND FUTURE WORK}

This paper deals with the landing phase of a vertical jump performed by a humanoid robot. We divided the landing motion pattern in two functions describing the relative feet/COM velocities and trajectories. One function (the pre-landing function) is aimed to reduce the feet/ground velocity at the moment of touching ground, in order to reduce the impact force and to obtain data of the state of the robot at landing time. These data are used by the second function (landing function) which adapts the actual state to the desired one. The motion pattern was simulated and showed satisfactory results in terms of ground reaction force.

To test the reliability of the pattern, some errors were implemented on the flight time and the momentum. The real flight time is difficult to evaluate as according to free flight motion equations. This time should start when the COM reaches the desired take-off velocity and finish when the robot motion stops. In our case, the flight time matches the moment the robot actually leaves the ground (when the GRF goes down to $20 \mathrm{~N}$ on each foot). The tests on error on flight times show that the forces registered by the sensors remained within feasible values for the sensors, for $t_{\mathrm{ld}}^{\mathrm{m}}=t_{\mathrm{ld}}^{\mathrm{th}} \pm 7 \%$.
Error on the momentum resulted in a huge value for the ankle torque sensor around $y$-axis. This situation cannot be faced in case of experimentation on HRP-2 robot. One next step of this study is then to cope with this particular situation. Another perspective is to compare different functions for jump pattern, such as trajectory optimization. Advantages of using such approach are to lead to more general dynamic motion generation, and to access a movement with low energy cost. A drawback is the impossibility to optimize trajectories while the motion is on, as time is an important factor in this study.

\section{ACKNOWLEDGMENT}

This research is supported by a post-doctoral Fellowship of Japan Society of Promotion for Science (JSPS), and by a Research Grant of JSPS.

\section{REFERENCES}

[1] K. Arikawa and T. Mita. Design of multi-dof jumping robot. In IEEE International Conference on Robotics and Automation (ICRA), pages 3992-3997, 2002.

[2] M. Gienger et al. Toward the design of a biped jogging robot. In IEEE International Conference on Robotics and Automation (ICRA), pages 4140-4145, 2001

[3] Y. Guan, K. Yokoi, N.E. Sian, and K. Tanie. Feasibility of humanoid robots stepping over obstacles. In IEEE/RSJ International Conference on Intelligent Robots and Systems (IROS), 2004.

[4] K. Hirai, M. Hirose, Y. Haikawa, and T. Takenaka. The development of honda humanoid robot. In IEEE International Conference on Robotics and Automation (ICRA), pages 1321-1326, 1998.

[5] S. Kajita, T. Nagasaki, K. Kaneko, K. Yokoi, and K. Tanie. A hop towards running humanoid biped. In IEEE International Conference on Robotics and Automation (ICRA), pages 629-635, 2004.

[6] J.H. Kim and J.H. Oh. Walking control of the humanoid platform khr-1 based on torque feedback control. In IEEE International Conference on Robotics and Automation (ICRA), pages 623-629, 2004.

[7] K. Nagasaka, Y. Kuroki, S. Suzuki, Y. Itoh, and J. Yamaguchi. Integrated motion control for walking, jumping and running on small bipedal entertainment robot. In IEEE International Conference on Robotics and Automation (ICRA), pages 3189-3194, 2004.

[8] K. Nishiwaki, T. Sugihara, S. Kagami, F. Kanehiro, M. Inaba, and H. Inoue. Design and development of research platform for perceptionaction integration in humanoid robot: H6. In IEEE/RSJ International Conference on Intelligent Robots and Systems (IROS), pages 1559$1564,2000$.

[9] V. Nunez and N. Nadjar-Gauthier. Control strategy for vertical jump of humanoid robots. In IEEE/RSJ International Conference on Intelligent Robots and Systems (IROS), 2005.

[10] Y. Ogura, H. Aikawa, H. Lim, and A. Takanishi. Development of a human-like walking robot. In IEEE International Conference on Robotics and Automation (ICRA), pages 134-140, 2004.

[11] R.R. Playter and M.H. Raibert. Control of a biped somersault in 3d. In IEEE/RSJ International Conference on Intelligent Robots and Systems (IROS), 1992.

[12] M.H. Raibert, Jr H.B. Brown, and M. Chepponis. Experiments in balance with a $3 \mathrm{~d}$ one-legged hopping machine. International Journal of Robotics Research, 3(2):75-92, 1984.

[13] S. Sakka and K. Yokoi. Humanoid vertical jumping based on force feedback and inertial forces optimization. In IEEE International Conference on Robotics and Automation (ICRA), 2005.

[14] Honda website. Asimo demonstration. http://world.honda.com/HDTV/ASIMO/. 